\title{
Charmed signatures for phase transitions in heavy-ion collisions
}

\section{Elena Bratkovskaya*}

Frankfurt Institute for Advanced Studies,

Ruth-Moufang-Str. 1, 60438 Frankfurt am Main, Germany

E-mail: Elena.Bratkovskaya@th.physik.uni-frankfurt.de

\section{Olena Linnyk}

Frankfurt Institute for Advanced Studies,

Ruth-Moufang-Str. 1, 60438 Frankfurt am Main, Germany

\section{Wolfgang Cassing}

Institut für Theoretische Physik,Universität Giessen,

Heinrich-Buff-Ring 16, 35392 Giessen, Germany

\section{Horst Stöcker}

Institut für Theoretische Physik, Johann Wolfgang Goethe University,

Max-von-Laue-Str. 1, 60438 Frankfurt am Main, Germany

Frankfurt Institute for Advanced Studies,

Ruth-Moufang-Str. 1, 60438 Frankfurt am Main, Germany

\begin{abstract}
The interplay of charmonium production and suppression in $I n+I n$ and $P b+P b$ reactions at $158 \mathrm{AGeV}$ and in $A u+A u$ reactions at $\sqrt{s}=200 \mathrm{GeV}$ is investigated with the HSD transport approach within the 'hadronic comover model' and the 'QGP melting scenario'. The results for the $J / \Psi$ suppression and the $\Psi^{\prime}$ to $J / \Psi$ ratio are compared to the recent data of the NA50, NA60, and PHENIX Collaborations. We find that, at $158 \mathrm{AGeV}$, the comover absorption model performs better than the scenario of abrupt threshold melting. However, neither interaction with hadrons alone nor simple color screening satisfactory describes the data at $\sqrt{s}=200 \mathrm{GeV}$. A deconfined phase is clearly reached at RHIC, but a theory having the relevant degrees of freedom in this regime (strongly interacting quarks/gluons) is needed to study its transport properties.
\end{abstract}

Critical Point and Onset of Deconfinement - 4th International Workshop July 9 - 13, 2007

Darmstadt, Germany

${ }^{*}$ Speaker. 


\section{Introduction}

Measurements of charmonium production in heavy-ion collisions at different energies can provide clear signatures of the onset of deconfinement. Indeed, according to potential model predictions and to the pioneering idea of Matsui and Satz [1], $c \bar{c}$ meson states might no longer be formed in a very hot fireball due to color screening [2, 3, 4]. This initially intuitive expectation has guided experimental studies for almost two decades. However, more recent lattice QCD calculations have shown that the $J / \Psi$ survives up to at least $1.5 T_{c}$ $\left(T_{c} \approx 170\right.$ to $185 \mathrm{MeV}$ ) such that the lowest $c \bar{c}$ states may remain bound up to rather high energy density $[5,6,7,8]$. On the other hand, the $\chi_{c}$ and $\Psi^{\prime}$ appear to melt soon above $T_{c}$.

According to present knowledge, the charmonium production in heavy-ion collisions, i.e. $c \bar{c}$ pairs, occurs exclusively at the initial stage of the reaction in primary nucleonnucleon collisions. At the very early stage color dipole states are expected to be formed ( $c f$. Refs. $[9,10])$. These $c \bar{c}$ states are assumed to be absorbed in a 'pre-resonance state' before the final hidden charm mesons are formed. Such absorption - denoted by 'normal nuclear suppression' - is also present in $p+A$ reactions and is determined by a dissociation cross section $\sigma_{B} \sim 4$ to $7 \mathrm{mb}$. Those charmonia or 'pre-resonance' states that survive normal nuclear suppression during the short overlap phase of the Lorentz contracted nuclei furthermore suffer from (i) a possible dissociation in the deconfined medium at sufficiently high energy density and (ii) the interactions with secondary hadrons (comovers) formed in a later stage of the nucleus-nucleus collision.

In the QGP 'threshold scenario', e.g the geometrical Glauber model of Blaizot et al. [11] as well as the percolation model of Satz [3], the QGP suppression '(i)' sets in rather abruptly as soon as the energy density exceeds a threshold value $\varepsilon_{c}$, which is a free parameter. This version of the standard approach is motivated by the idea that the charmonium dissociation rate is drastically larger in a quark-gluon-plasma (QGP) than in a hadronic medium [3]. On the other hand, the extra suppression of charmonia in the high density phase of nucleus-nucleus collisions at SPS energies $[12,13,14,15]$ has been attributed to inelastic comover scattering ( $c f .[10,16,17,18,19,20,21,22,23]$ and Refs. therein) assuming that the corresponding $J / \Psi$-hadron cross sections are in the order of a few $m b[24,25,26,27]$. In these models 'comovers' are viewed not as asymptotic hadronic states in vacuum but rather as hadronic correlators (essentially of vector meson type) that might well survive at energy densities above $1 \mathrm{GeV} / \mathrm{fm}^{3}$. Additionally, alternative absorption mechanisms might play a role, such as gluon scattering on color dipole states as suggested in Refs. [28, 29, 30, 31] or charmonium dissociation in the strong color fields of overlapping strings [32].

We recall that apart from absorption or dissociation channels for charmonia also recombination channels such $D+\bar{D} \rightarrow X_{c}+$ meson $\left(X_{c}=\left(J / \Psi, \chi_{c}, \Psi^{\prime}\right)\right)$ play a role in the hadronic phase. These backward channels - relative to charmonium dissociation with comoving mesons - have been found to be practically negligible at the SPS energies [33], but extremely important at the top RHIC energy of $\sqrt{s}=200 \mathrm{GeV}$ [34]. This is in accordance with independent studies in Refs. [26, 29, 35, 36] and earlier analysis within the HSD transport approach [37, 38].

The explicit treatment of initial $c \bar{c}$ production by primary nucleon-nucleon collisions and the implementation of the comover model - involving a single matrix element $M_{0}$ fixed 
by the data at SPS energies - as well as the QGP threshold scenario in HSD were explained in Ref. [33] (see Fig. 1 of Ref. [33] for the relevant cross sections). We recall that the 'threshold scenario' for charmonium dissociation is implemented as follows: whenever the local energy density $\varepsilon(x)$ is above a threshold value $\varepsilon_{j}$ (where the index $j$ stands for $J / \Psi, \chi_{c}, \Psi^{\prime}$ ), the charmonium is fully dissociated to $c+\bar{c}$. The default threshold energy densities adopted are $\varepsilon_{1}=16 \mathrm{GeV} / \mathrm{fm}^{3}$ for $J / \Psi, \varepsilon_{2}=2 \mathrm{GeV} / \mathrm{fm}^{3}$ for $\chi_{c}$, and $\varepsilon_{3}=2 \mathrm{GeV} / \mathrm{fm}^{3}$ for $\Psi^{\prime}$.

It is presently not clear, if also the $D$-mesons survive at temperatures $T>T_{c}$, but strong correlations between a light quark (antiquark) and a charm antiquark (quark) are likely to persist [39]. One may also speculate that similar correlations survive also in the light quark sector above $T_{c}$ such that 'hadronic comovers' - most likely with different spectral functions - might show up also at energy densities above $1 \mathrm{GeV} / \mathrm{fm}^{3}$, which is taken as a characteristic scale for the critical energy density. Therefore, we study both possibilities: with and without comover absorption (and $D+\bar{D}$ recombination) at energy densities above the cut-energy density parameter $\varepsilon_{\text {cut }}=1 \mathrm{GeV} / \mathrm{fm}^{3}$.

Since we aimed to answer, whether the charmonium dissociation mechanism is identical at SPS and top RHIC energies, we adopted in [34] the same cross sections for the colordipole dissociation with nucleons as well the dissociation cross sections with comovers as in Ref. [33] for SPS. Consequently no free parameters entered our studies at the RHIC energy. We note that the hadronic comover reactions for the recreation of charmonia $J / \Psi, \chi_{c}, \Psi^{\prime}$ by $D+\bar{D}$ reactions are incorporated in all simulations. This is a 'default' in the comover absorption and recreation scenario and 'necessary' in the QGP 'threshold scenario' because (in view of Fig. 4, 1.h.s.) practically all charmonia are dissolved due to the very high initial energy densities. Therefore, any model without recreation of charmonia is clearly ruled out by the PHENIX data.

\section{Comparison to data}

We directly step on with results for the charmonium suppression at SPS energies in comparison with the experimental data from the NA50 and NA60 Collaborations. These Collaborations present their results on $J / \Psi$ suppression as the ratio of the dimuon decay of $J / \Psi$ relative to the Drell-Yan background from $2.9-4.5 \mathrm{GeV}$ invariant mass as a function of the transverse energy $E_{T}$, or alternative, as a function of the number of participants $N_{\text {part }}$, i.e.

$$
B_{\mu \mu} \sigma(J / \Psi) /\left.\sigma(D Y)\right|_{2.9-4.5},
$$

where $B_{\mu \mu}$ is the branching ratio for $J / \Psi \rightarrow \mu^{+} \mu^{-}$. In order to compare our results to experimental data, we need an extra input, i.e. the normalization factor $B_{\mu \mu} \sigma_{N N}(J / \Psi) / \sigma_{N N}(D Y)$, which defines the $J / \Psi$ over Drell-Yan ratio for elementary nucleon-nucleon collisions. We choose $B_{\mu \mu} \sigma_{N N}(J / \Psi) / \sigma_{N N}(D Y)=36$ in line with the NA60 compilation [15].

Furthermore, the $\Psi^{\prime}$ suppression is presented experimentally by the ratio

$$
\frac{B_{\mu \mu}\left(\Psi^{\prime} \rightarrow \mu \mu\right) \sigma\left(\Psi^{\prime}\right) / \sigma(D Y)}{B_{\mu \mu}(J / \Psi \rightarrow \mu \mu) \sigma(J / \Psi) / \sigma(D Y)} .
$$

In our calculations we adopt this ratio to be 0.0165 for nucleon-nucleon collisions, which is again based on the average over $p p, p d, p A$ reactions [42]. 


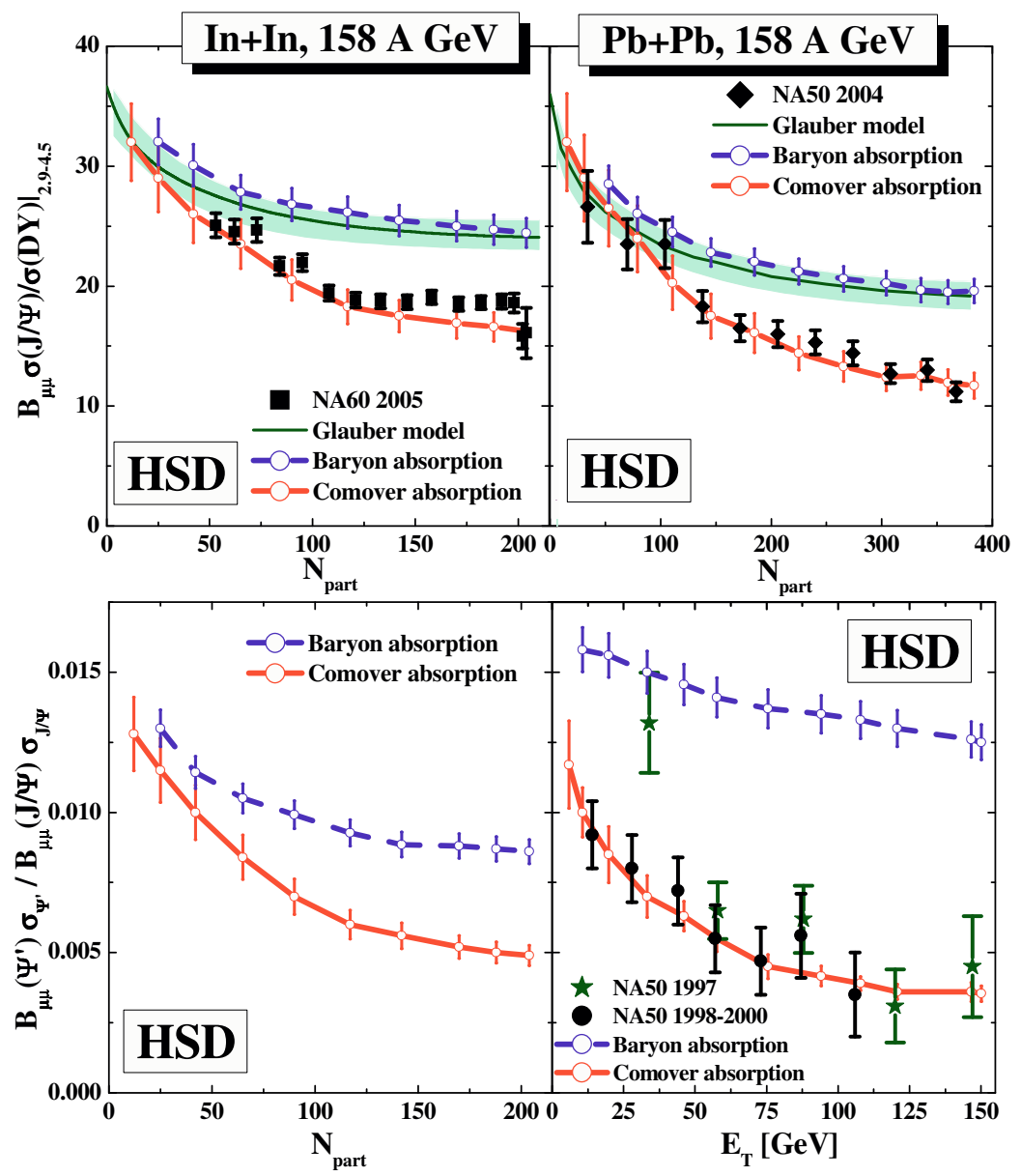

Figure 1: The ratio $B_{\mu \mu} \sigma(J / \Psi) / \sigma(D Y)$ as a function of the number of participants in In+In (1.h.s.) and $\mathrm{Pb}+\mathrm{Pb}$ reactions (r.h.s.) at $158 \mathrm{~A} \cdot \mathrm{GeV}$. The full symbols denote the data from the NA50 and NA60 Collaborations (from Refs. [40, 15, 41]), while the dashed (blue) lines represent the HSD calculations including only dissociation channels with nucleons. The lower parts of the figure show the HSD results in the same limit for the $\Psi^{\prime}$ to $J / \Psi$ ratio as a function of $N_{\text {part }}$ (for In+In) or the transverse energy $E_{T}$ (for $\mathrm{Pb}+\mathrm{Pb}$ ). The solid (red) lines show the HSD results for the comover absorption model with a matrix element squared $\left|M_{0}\right|^{2}=0.18 \mathrm{fm}^{2} / \mathrm{GeV}^{2}$. The (light blue) bands in the upper parts of the figure give the estimate for the normal nuclear $J / \Psi$ absorption as calculated by the NA60 Collaboration. The vertical lines on the graphs reflect the theoretical uncertainty due to limited statistics of the calculations. The figure is taken from [33].

We first show in Fig. 1 the calculated ratio (2.1) as a function of $N_{\text {part }}$ for $\mathrm{Pb}+\mathrm{Pb}$ and $\mathrm{In}+\mathrm{In}$ collisions at $158 \mathrm{~A} \cdot \mathrm{GeV}$ (upper plots) in the nuclear suppression scenario, i.e. without comover dissociation or 'QGP threshold suppression'. The dashed (blue) lines stand for the HSD result while the (light blue) bands give the estimate for the normal nuclear $J / \Psi$ absorption as calculated by the NA60 Collaboration. The normal nuclear suppression from HSD is seen to be slightly lower than the (model dependent) estimate from NA60, however, agrees quite well with their model calculations for more central reactions. The various experimental data points have been taken from Refs. [15, 40, 41]. As a next step we add the comover dissociation channels within the model described in [33] for a matrix 


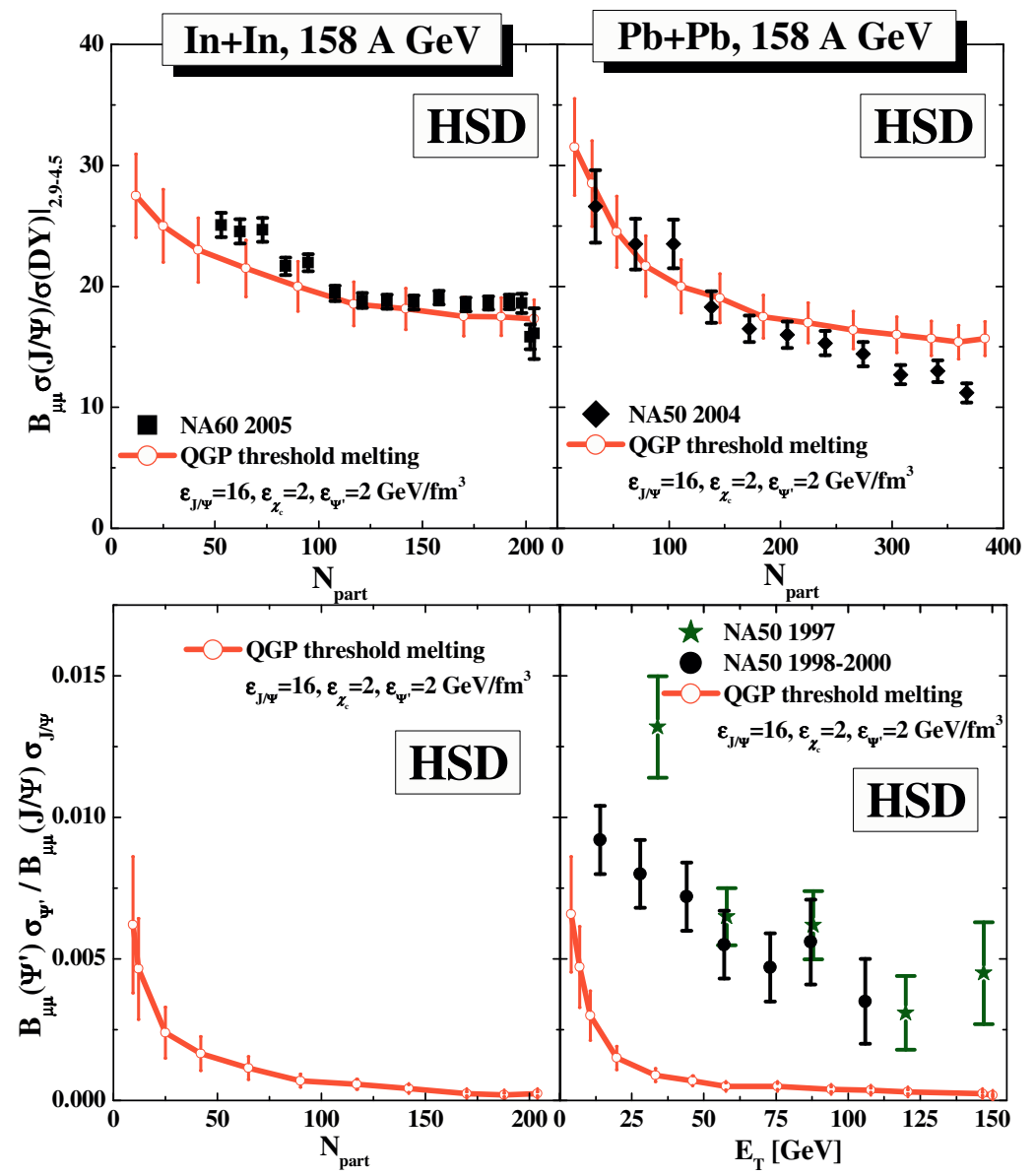

Figure 2: Same as Fig. 1 but for the 'QGP threshold scenario' with $\varepsilon_{J / \Psi}=16 \mathrm{GeV} / \mathrm{fm}^{3}, \varepsilon_{\chi_{c}}=2$ $\mathrm{GeV} / \mathrm{fm}^{3}=\varepsilon_{\Psi}$ ' while discarding comover absorption. The figure is taken from [33].

element squared $\left|M_{0}\right|^{2}=0.18 \mathrm{fm}^{2} / \mathrm{GeV}^{2}$. Note that in this case the charmonium reformation channels are incorporated, too, but could be discarded since the charmonium regeneration is negligible at SPS energies (cf. Ref. [37]). The extra suppression of charmonia by comovers is seen in Fig. 1 (solid red lines) to match the $J / \Psi$ suppression in $\mathrm{In}+\mathrm{In}$ and $\mathrm{Pb}+\mathrm{Pb}$ as well as the $\Psi^{\prime}$ to $J / \Psi$ ratio (for $\mathrm{Pb}+\mathrm{Pb}$ ) rather well. The more recent data (1998-2000) for the $\Psi^{\prime}$ to $J / \Psi$ ratio agree with the HSD prediction within error bars. This had been a problem in the past when comparing to the 1997 data (dark green stars). The $\Psi^{\prime}$ to $J / \Psi$ ratio for In+In versus centrality is not yet available from the experimental side but the theoretical predictions are provided in Fig. 1 and might be approved/falsified in near future.

The results for the 'threshold scenario' are displayed in Fig. 2 in comparison to the same data for the thresholds $\varepsilon_{J / \Psi}=16 \mathrm{GeV} / \mathrm{fm}^{3}, \varepsilon_{\chi_{c}}=2 \mathrm{GeV} / \mathrm{fm}^{3}=\varepsilon_{\Psi^{\prime}}$. In this scenario the $J / \Psi$ suppression is well described for In+In but the suppression is slightly too weak for very central $\mathrm{Pb}+\mathrm{Pb}$ reactions. This result emerges since practically all $\chi_{c}$ and $\Psi^{\prime}$ dissolve for $N_{\text {part }}>100$ in both systems whereas the $J / \Psi$ itself survives at the energy densities reached in the collision. Since the nucleon dissociation is a flat function of $N_{\text {part }}$ for central reactions, the total absorption strength is flat, too. The deviations seen in Fig. 2 might indicate a partial melting of the $J / \Psi$ for $N_{\text {part }}>250$, which is not in line with most lattice QCD calculations 

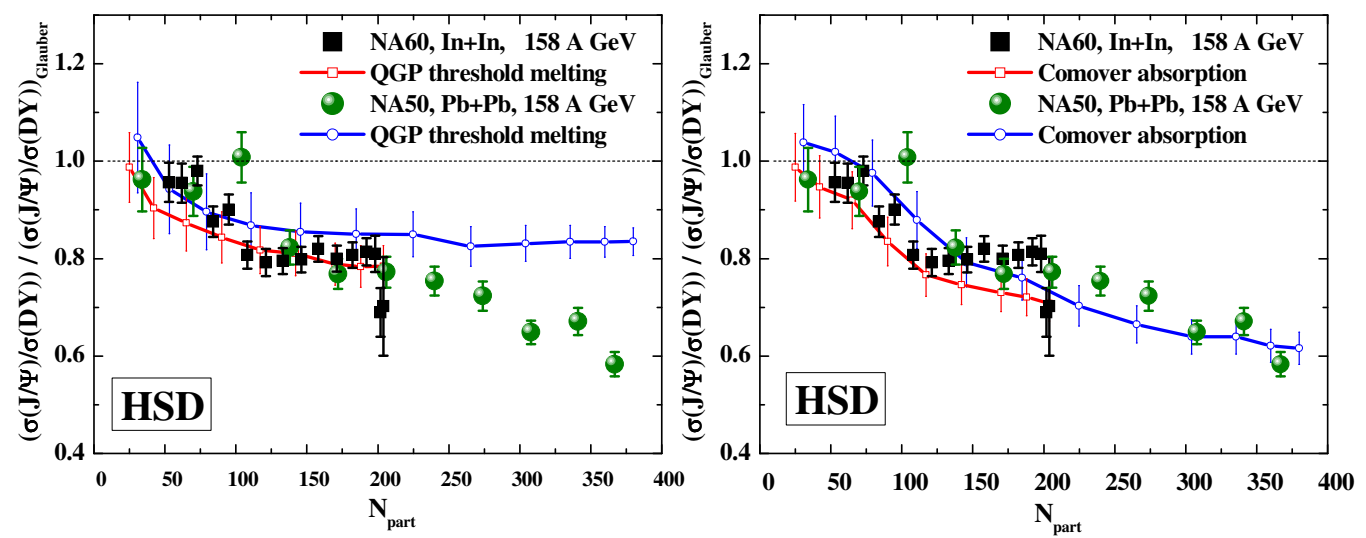

Figure 3: The ratio $B_{\mu \mu} \sigma(J / \Psi) / \sigma(D Y)$ as a function of the number of participants $N_{\text {part }}$ in In+In (red line with open squares) and $\mathrm{Pb}+\mathrm{Pb}$ reactions (blue line with open circles) at $158 \mathrm{~A} \cdot \mathrm{GeV}$ relative to the normal nuclear absorption given by the straight black line. The full dots and squares denote the respective data from the NA50 and NA60 Collaborations. The model calculations reflect the comover absorption model (right part) and the 'QGP threshold scenario' (left part) with $\varepsilon_{J / \Psi}=16$ $\mathrm{GeV} / \mathrm{fm}^{3}, \varepsilon_{\chi_{c}}=2 \mathrm{GeV} / \mathrm{fm}^{3}, \varepsilon_{\Psi^{\prime}}=6.55 \mathrm{GeV} / \mathrm{fm}^{3}$ while discarding comover absorption. Figure is taken from [33].

claiming at least $\varepsilon_{J / \Psi}>5 \mathrm{GeV} / \mathrm{fm}^{3}$. In fact, a lower threshold of $5 \mathrm{GeV} / \mathrm{fm}^{3}$ (instead of 16 $\mathrm{GeV} / \mathrm{fm}^{3}$ ) for the $J / \Psi$ has practically no effect on the results shown in Fig. 2. Furthermore, a threshold energy density of $2 \mathrm{GeV} / \mathrm{fm}^{3}$ for the $\Psi^{\prime}$ leads to a dramatic reduction of the $\Psi^{\prime}$ to $J / \Psi$ ratio which is in severe conflict with the data (lower part of Fig. 2). Also note that there is no step in the suppression of $J / \Psi$ versus centrality. As pointed out before by Gorenstein et al. in Ref. [43], this is due to energy density fluctuations in reactions with fixed $N_{\text {part }}$ (or $E_{T}$ ).

Additionally, one can plot the results in an intuitive though model-dependent way, as a ratio of the measured $J / \Psi$ yield divided by the normal nuclear absorption result calculated in the Glauber model. Since the NA60 Collaboration prefers to represent their data in this form, we additionally show in Fig. 3 our calculations for In+In (red lines with open squares) and $\mathrm{Pb}+\mathrm{Pb}$ (blue lines with open circles) as a function of the number of participants $N_{\text {part }}$ relative to the normal nuclear absorption given by the straight black line ${ }^{1}$. The full dots and squares denote the respective data from the NA50 and NA60 Collaborations. The model calculations reflect the comover absorption model (right part) and the 'QGP threshold scenario' (left part) with $\varepsilon_{J / \Psi}=16 \mathrm{GeV} / \mathrm{fm}^{3}, \varepsilon_{\chi_{c}}=2 \mathrm{GeV} / \mathrm{fm}^{3}, \varepsilon_{\Psi^{\prime}}=6.55 \mathrm{GeV} / \mathrm{fm}^{3}$. Since only the representation is different the message stays the same: The comover absorption model follows slightly better the fall of the $J / \Psi$ survival probability with increasing centrality whereas the 'threshold scenario' leads to an approximate plateau in both reactions for high centrality.

Let us now move to a much higher energy scale by calculating charmonium dynam-

\footnotetext{
${ }^{1}$ Note that recently the NA60 collaboration has refitted the parameters of their Galuber model, therefore newer data releases [44] might appear to be up- or down-scaled compared to the data plotted here [15], if shown in this particular representation (measured to expected ratio). This scaling falls within the systematic uncertainty of the ratio and does not change results and conclusions of our study.
} 


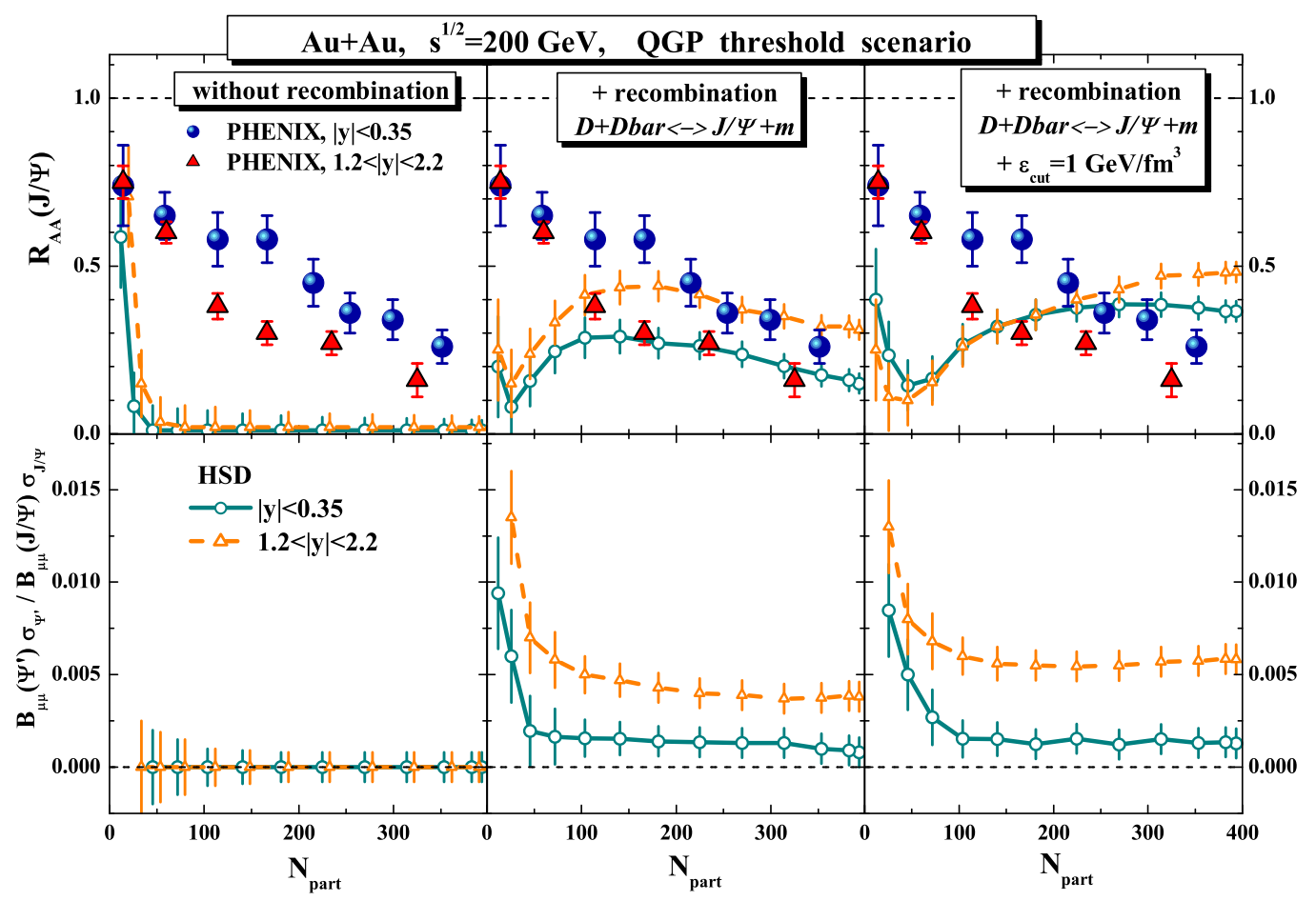

Figure 4: The $J / \Psi$ nuclear modification factor $R_{A A}$ for $A u+A u$ collisions at $\sqrt{s}=200 \mathrm{GeV}$ as a function of the number of participants $N_{\text {part }}$ in comparison to the data from [10] for midrapidity (full circles) and forward rapidity (full triangles). HSD results for the QGP 'threshold melting' scenarios are displayed in terms of the lower (green solid) lines for midrapidity $J / \Psi$ 's $(|y| \leq 0.35$ ) and in terms of the upper (orange dashed) lines for forward rapidity $(1.2 \leq y \leq 2.2$ ) within different recombination scenarios (see text). The error bars on the theoretical results indicate the statistical uncertainty due to the finite number of events in the HSD calculations. Predictions for the ratio $B_{\mu \mu}\left(\Psi^{\prime}\right) \sigma_{\Psi^{\prime}} / B_{\mu \mu}(J / \Psi) \sigma_{J / \Psi}$ as a function of the number of participants $N_{\text {part }}$ are shown in the lower set of plots. The figure is taken from [34].

ics at the top RHIC energy of $\sqrt{s}=200 \mathrm{GeV}$. In the initial stages of $A u+A u$ collisions at this $\sqrt{s}$, energy densities above $30 \mathrm{GeV} / \mathrm{fm}^{3}$ are reached [34]. Therefore, in the threshold melting scenario, all initially created $J / \Psi, \Psi^{\prime}$ and $\chi_{c}$ mesons melt. However, the PHENIX collaboration has found that at least $20 \%$ of $J / \Psi$ do survive at RHIC [45]. Thus, the importance of charmonium recreation is shown again. We account for $J / \Psi$ recreation via the $D \bar{D}$ annihilation processes as explained in detail in [33,34]. Note that in our approach, the cross sections of charmonium recreation in $D+\bar{D} \rightarrow J / \Psi+$ meson processes is fixed by detailed balance from the comover absorption cross section $J / \Psi+$ meson $\rightarrow D+\bar{D}$. But even after both these processes are added to the threshold melting mechanism, the centrality dependence of the $R_{A A}(J / \Psi)$ cannot be reproduced, especially in the peripheral collisions (see Fig. 4). This holds for both possibilities: with (r.h.s. of Fig. 4) and without (center of Fig. 4) the energy density cut $\varepsilon_{\text {cut }}$, below which $D$-mesons and comovers exist and can participate in $D+\bar{D} \leftrightarrow J / \Psi+$ meson reactions. 


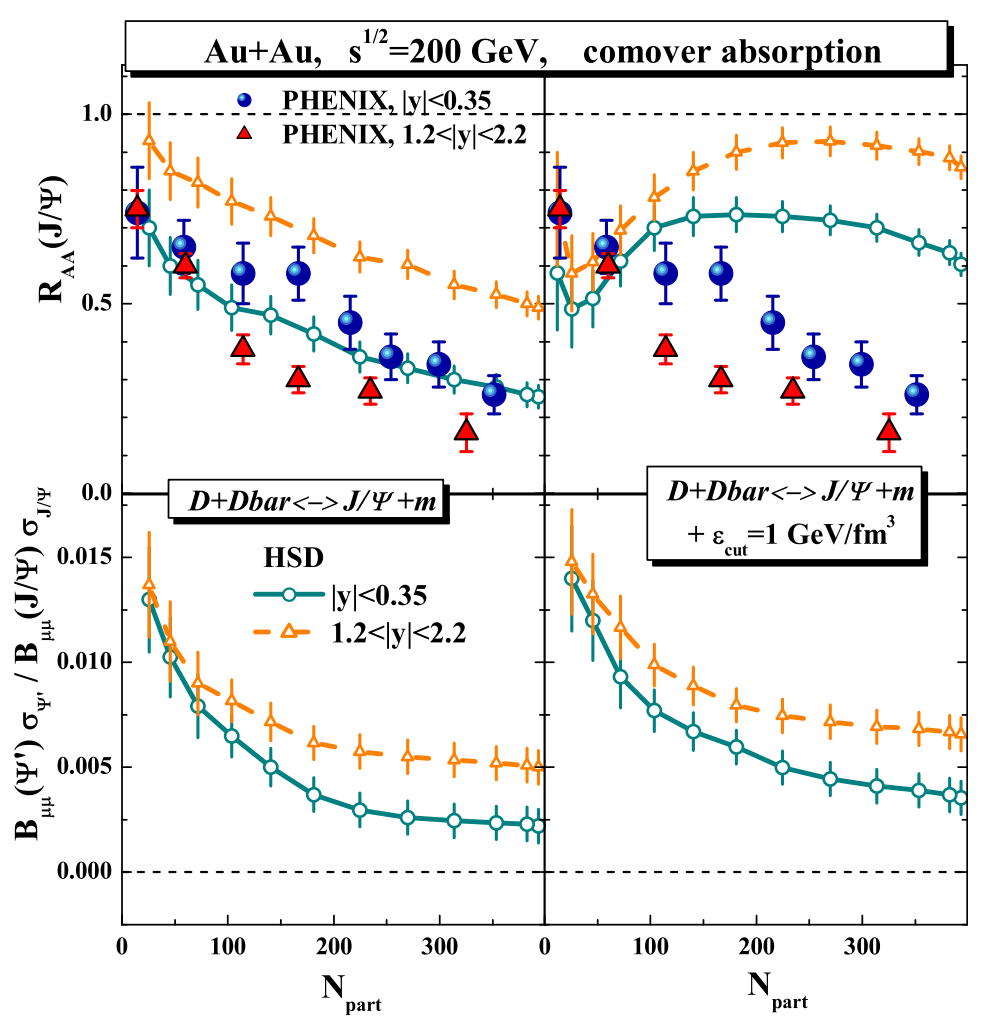

Figure 5: Same as Fig. 4 for the 'comover absorption scenario' including the charmonium reformation channels without cut in the energy density (l.h.s.) and with a cut in the energy density $\varepsilon_{c u t}=1 \mathrm{GeV} / \mathrm{fm}^{3}$ (see text for details). The figure is taken from [34].

We recall that the nuclear modification factor $R_{A A}$ is given by

$$
R_{A A}=\frac{d N(J / \Psi)_{A A} / d y}{N_{\text {coll }} \cdot d N(J / \Psi)_{p p} / d y},
$$

where $d N(J / \Psi)_{A A} / d y$ denotes the final yield of $J / \Psi$ in $A A$ collisions, $d N(J / \Psi)_{p p} / d y$ is the yield in elementary $p p$ reactions, $N_{\text {coll }}$ is the number of binary collisions.

Comover absorption scenarios give generally a correct dependence of the yield on the centrality. If an existence of D-mesons at energy densities above $1 \mathrm{GeV} / \mathrm{fm}^{3}$ is assumed, the amplitude of suppression of $J / \Psi$ at mid-rapidity is also well reproduced (see the line for 'comover without $\varepsilon_{\text {cut }}$ ' scenario in Fig.5, 1.h.s.). Note that this line correspond to the prediction made in the HSD approach in [38]. On the other hand, the rapidity dependence of the comover result is wrong, both with and without $\varepsilon_{c u t}$. If hadronic correlators exist only at $\varepsilon<\varepsilon_{c u t}$, comover absorption is insufficient to reproduce the $J / \Psi$ suppression even at mid-rapidity (see Fig. 5, r.h.s.). The difference between the theoretical curves marked 'comover $+\varepsilon_{\text {cut }}$ ' and the data shows the maximum supression that can be attributed to a deconfined medium.

\section{Summary}

We have investigated the formation and suppression dynamics of $J / \Psi, \chi_{c}$ and $\Psi^{\prime}$ mesons within the HSD transport approach for $I n+I n$ and $P b+P b$ reactions at $158 \mathrm{AGeV}$ 
and for $A u+A u$ reactions at $\sqrt{s}=200 \mathrm{GeV}$. Two currently discussed models, i.e. the 'hadronic comover absorption and reformation' model as well as the 'QGP threshold melting scenario' have been compared to the available experimental data. We adopted the same parameters for cross sections (matrix elements) or threshold energies at both bombarding energies.

We find that both scenarios are compatible with experimental observation of $J / \Psi$ suppression at SPS energies, while the $\Psi^{\prime}$ to $J / \Psi$ ratio data appear to be in conflict with the 'threshold melting' scenario [33]. On the other hand, both 'comover absorption' and 'threshold melting' fail severely at RHIC energies [34]. The failure of the 'hadronic comover absorption' model goes in line with its underestimation of the collective flow $v_{2}$ of leptons from open charm decay as investigated in Ref. [46]. This suggests that 1) a deconfined phase is clearly reached at RHIC, 2) the dynamics of $c, \bar{c}$ quarks at this energy are dominated by partonic interactions in the strong QGP (sQGP) which cannot be modeled by 'hadronic' interactions or described appropriately by color screening alone.

\section{Acknowledgement}

The authors acknowledge financial support by BMBF.

\section{References}

[1] T. Matsui and H. Satz, Phys. Lett. B178, 416 (1986).

[2] H. Satz, Rep. Progr. Phys. 63, 1511 (2000).

[3] H. Satz, J. Phys. G32, R25 (2006).

[4] F. Karsch, D. Kharzeev, and H. Satz, Phys. Lett. B637, 75 (2006), hep-ph/0512239.

[5] G. Aarts, C. Allton, M. B. Oktay, M. Peardon, and J.-I. Skullerud, (2007), arXiv:0705.2198[hep-lat].

[6] S. Datta, F. Karsch, P. Petreczky, and I. Wetzorke, J. Phys. G30, S1347 (2004).

[7] M. Asakawa and T. Hatsuda, J. Phys. G30, S1337 (2004).

[8] F. Karsch, J. Phys. G30, S887 (2004).

[9] D. Kharzeev, C. Lourenco, M. Nardi, and H. Satz, Z. Phys. C74, 307 (1997), hep-ph/9612217.

[10] N. Armesto and A. Capella, Phys. Lett. B430, 23 (1998), hep-ph/9705275.

[11] J. P. Blaizot and J. Y. Ollitrault, Phys. Rev. Lett. 77, 1703 (1996), hep-ph/9606289.

[12] NA50, M. C. Abreu et al., Phys. Lett. B410, 337 (1997).

[13] NA50, M. C. Abreu et al., Phys. Lett. B477, 28 (2000).

[14] NA50, M. C. Abreu et al., Phys. Lett. B450, 456 (1999).

[15] NA60, A. Foerster et al., J. Phys. G32, S51 (2006), nucl-ex/0609039.

[16] W. Cassing and E. L. Bratkovskaya, Nucl. Phys. A623, 570 (1997).

[17] W. Cassing and E. L. Bratkovskaya, Phys. Rep. 308, 65 (1999).

[18] R. Vogt, Phys. Rep. 310, 197 (1999). 
[19] C. Gerschel and J. Hüfner, Ann. Rev. Nucl. Part. Sci. 49, 255 (1999).

[20] W. Cassing, E. L. Bratkovskaya, and S. Juchem, Nucl. Phys. A674, 249 (2000).

[21] D. E. Kahana and S. H. Kahana, Prog. Part. Nucl. Phys. 42, 269 (1999).

[22] C. Spieles et al., J. Phys. G25, 2351 (1999), Phys. Rev. C60 (1999) 054901.

[23] L. Gerland, L. Frankfurt, M. Strikman, H. Stöcker, and W. Greiner, Nucl. Phys. A663, 1019 (2000).

[24] K. L. Haglin, Phys. Rev. C61, 031903 (2000).

[25] Z. Lin and C. M. Ko, Phys. Rev. C62, 034903 (2000).

[26] Z. Lin and C. M. Ko, J. Phys. G27, 617 (2001).

[27] A. Sibirtsev, K. Tsushima, and A. W. Thomas, Phys. Rev. C63, 044906 (2001).

[28] B. Zhang, C. M. Ko, B.-A. Li, Z. Lin, and B.-H. Sa, Phys. Rev. C62, 054905 (2000).

[29] L. Grandchamp and R. Rapp, Phys. Lett. B523, 60 (2001), Nucl. Phys. A 709 (2002) 415.

[30] D. Blaschke, Y. Kalinovsky, and V. Yudichev, Lect. Notes Phys. 647, 366 (2004), hep-ph/0410338.

[31] M. Bedjidian et al., hep-ph/0311048.

[32] J. Geiss, C. Greiner, E. L. Bratkovskaya, W. Cassing, and U. Mosel, Phys. Lett. B447, 31 (1999).

[33] O. Linnyk, E. L. Bratkovskaya, W. Cassing, and H. Stöcker, Nucl. Phys. A786, 183 (2007).

[34] O. Linnyk, E. L. Bratkovskaya, W. Cassing, and H. Stöcker, arXiv:0705.4443[nucl-th].

[35] A. Andronic, P. Braun-Munzinger, K. Redlich, and J. Stachel, Nucl. Phys. A789, 334 (2007).

[36] A. Andronic, P. Braun-Munzinger, K. Redlich, and J. Stachel, (2007), arXiv:0708.1488[nucl-th].

[37] E. L. Bratkovskaya, W. Cassing, and H. Stöcker, Phys. Rev. C67, 054905 (2003).

[38] E. L. Bratkovskaya, A. P. Kostyuk, W. Cassing, and H. Stöcker, Phys. Rev. C69, 054903 (2004).

[39] H. van Hees and R. Rapp, Phys. Rev. C71, 034907 (2005).

[40] NA50, B. Alessandro et al., Eur. Phys. J. C39, 335 (2005), hep-ex/0412036.

[41] NA50, B. Alessandro et al., nucl-ex/0612013.

[42] NA50, B. Alessandro et al., Phys. Lett. B553, 167 (2003).

[43] M. I. Gorenstein, A. P. Kostyuk, H. Stöcker, and W. Greiner, Phys. Lett. B509, 277 (2001).

[44] NA60, R. Arnaldi et al., (2007), arXiv:0706.4361 [nucl-ex].

[45] PHENIX, H. Büsching et al., Nucl. Phys. A774, 103 (2006).

[46] E. L. Bratkovskaya, W. Cassing, H. Stöcker, and N. Xu, Phys. Rev. C71, 044901 (2005). 